\title{
Experimental Study of the Ventilation Arrangement's Effect on Particle Concentration in a Surgery Room
}

\author{
Ali Parcheforosh ${ }^{1}$, Arash Mousemi ${ }^{1}$, Sorour A. Alotaibi ${ }^{2}$, Alireza Khademi ${ }^{3}$ \\ ${ }^{1}$ Department of Mechanical Engineering, Sharif University of Technology \\ Azadi Ave, Tehran, Iran \\ aliparcheforosh@gmail.com; arashmousemi@gmail.com \\ ${ }^{2}$ Mechanical Engineering Department, College of Engineering and Petroleum, Kuwait University \\ Fourth Ring Rd, Kuwait City, Kuwait \\ sr.alotaibi@ku.edu.kw \\ ${ }^{3}$ Department of Aerospace Engineering, Sharif University of Technology \\ Azadi Ave, Tehran, Iran \\ khademi_alireza@ae.sharif.edu
}

\begin{abstract}
Hospital ventilation is defined as the providing of fresh and clean air stream in hospital wards or patients' room to provide and establish a healthy environment for personnel's and patient's breath, reducing concentration of produced contaminations in hospital and removing them from clean spaces. Thus, it can prevent spread of airborne infections among patients, people who works around and more importantly, outside the hospital. Hospital infections have a huge influence on mortality of the patients in which respiratory tract infections and surgical wound infections plays a major role. Depending on the quality and the mass flow rate of the exploited air, cleanness of a surgery room can be achieved by supplying an adequate amount of air. In this regard, arrangement of inlet and outlet gates have the most considerable impact among geometrical parameters. However, it should be mentioned that positioning of the outlet gates has negligible influence on the distribution of stream properties like velocity, temperature and concentration of the contaminations relative to inlet gates. In this study, the concentration of aerosols for different dimensions and for different modes of inlet gates in a completely equipped surgery room is investigated and the average particle concentration of them is compared. It can be illustrated that obtained results changes with variation of particles' dimensions.
\end{abstract}

Keywords: Particle Concentration, Surgery Room, Ventilation Arrangement, Aerosols.

\section{Introduction}

Bacterial transmission in air is the most essential factor in surgical site infections. According to the announcement of the Center of Disease Control (CDC), surgical site infections are among the first three important factors of hospital infections, which involve 14 up to $16 \%$ of them [1]. Being estimated in 1999, a single surgical infection for each patient can make them stay for 10 days longer, which means a 5000\$ increase in expenditures[2]. Surgical infections can be produced up to 30 days after the surgery and they can be classified into three groups [3]: superficial incisional, deep incisional and organ or space infections. Practical improvement in infection control has been made like enhancing ventilation of the surgery room, sterilization of the room and the equipment and also exploiting new techniques to make surgery less dangerous. Depending on patient, personnel, type of surgery and environmental conditions, systematic but practical methods are needed for reducing the infections risks. Moreover, Staphylococcus aureus is known as the most important bacteria in surgical infections which has been effective on the wide range of the infections[4]. Live on humans' body skin, dead skin cells are the main source of producing these bacteria. It is estimated that approximately 1000 particles carrying bacteria can be separated from surgeon's body and released in the room [5]. Furthermore, distance between surgeon's body and patient's body can play a significant role on the possibility of infection. In the past decade, many different practical methods were investigated for removing surgery room's bacteria, which the most effective one is fast transmission of bacteria by a suitable air stream in the room. In this method [6], Particulate matter from the respiratory system can be guided into output gates swiftly by producing a welldirected stream and by reducing circulation in the air specially around patient, bacteria penetration can be restricted. 
In many studies particles' diameter which can carry bacteria are considered between 5 to $10 \mu \mathrm{m}$ in which gravity and buoyancy force have a huge influence on the particles [7]. In this study, concentration of particles in the air for different dimensions, with the complete internal room layout, for different arrangement of inlet gates is investigated. In this regard, four gates arrangements are studied which are diagonal, lateral, central and full open gates. Based on the result of Balaras et al [8], temperature and the relative humidity is considered $28^{\circ} \mathrm{C}$ and $35 \%$, respectively. Optimum air velocity in the inlet gate is $0.15 \mathrm{~m} / \mathrm{s}$ based on Memarzadeh et al [9], However, the inlet velocity is considered $0.2 \mathrm{~m} / \mathrm{s}$ in this case.

\section{Test Case}

Being equipped and designed like many conventional surgery rooms, clean room of the Sharif University of Technology is considered as a test case for this investigation. The room has dimensions of $2.9 \times 3.7$ square meters while it is heighted 2.5 meters. Moreover, having six inlet gates in the ceiling each of them dimensioned $60 \times 60$ square centimetres, the effect of different arrangements of inlet gates can be studied. Additionally, the selected room has five outlet gates on the walls having dimensions of $30 \times 45$ square centimetres each. Furthermore, one of the important factors for achieving a specific level of cleanness and eliminating the circulated streams is removing corners and generally dead pockets in which particles can maintain in these areas due to turbulence streams. In this highly particle concentrated zones, high momentum air can enter and carry a huge amount of particles with them to the main air stream which can increase the air pollution around the patient. Fortunately, this principle is taken into account in the construction of the studied cleanroom to an extent that no corner and sharp edges can be seen in the cleanroom.

\section{Results}

Choosing the experiment type can be influenced by parameters like room's design, related usage and needed pureness level. Three different conditions can be considered for the classification of cleanrooms according to the IEST standard before the concentration test. Accordingly, cleanrooms can be assorted into built cleanrooms, rest cleanrooms and the operational ones. First group is applied to the ones that the whole room with full equipment are ready to work but equipment and working stuff are not present in the room while in the second group just personnel are not present and the operational group is referred to ones in which both equipment and personnel are present. Considering the mentioned classification and the test case, built mode is suitable for this investigation. In this part, Concentration of particles with different dimensions for various inlet gates layout is investigated in a room with complete equipment. Arrangements like diagonal, central and lateral are used regularly in cleanrooms, however, a study on full open gates layout is performed additional to the others. Fig. 1 shows the particle concentration for each particle dimension with respect to the exploited layout and has the most consistency with expectations. Having short distance between inlet and outlet gates in a way that air stream does not have enough time for moving the particles, lateral arrangement has more deposited particles among the others. Regarding to the mentioned argument, although, central arrangement should leave more particles than the lateral one, it can be illustrated that central arrangement has left fewer particles in the room. This is firstly due to errors in particle counter device and secondly because of forces acting on the particle which can be classified as follows [5]: gravity force, buoyancy force, Brownian motion force and the Thermophoretic forces being resulted from scalar gradients like temperature. Table 1 shows order of magnitude among the mentioned forces.

Table 1:The order of magnitude of the different forces relative to the force of gravity in terms of particle size [10].

\begin{tabular}{|c|c|c|c|}
\hline \multirow{2}{*}{ Force(N) } & \multicolumn{3}{|c|}{ Particle dimension $(\mu \mathrm{m})$} \\
\cline { 2 - 4 } & $10^{-1}$ & 1 & 10 \\
\hline Gravity & 1 & 1 & 1 \\
\hline Buoyancy & $10^{-2}$ & $10^{-3}$ & $10^{-3}$ \\
\hline Brownian & $10^{0.5}$ & $10^{-2}$ & $10^{-5}$ \\
\hline Thermophoretic & 1 & $10^{-2}$ & $10^{-4}$ \\
\hline
\end{tabular}


Having maximum area for removing particles, full open gates arrangement is the best choice which can be conspicuously illustrated from Fig. 1(a). Furthermore, Fig. 1(b) shows apart from the fact that diagonal layout was the worst case among others, in other cases, obtained results did not match with the expectations such as the superiority of full open gates arrangement over the others. This phenomenon can be explained by taking following reasons into consideration. Firstly, according to Fig. 1(b), measuring equipment may not count particle numbers meticulously as its error can be increased as a result of particle dimensions which are too small between 0.3 up to $0.5 \mu \mathrm{m}$ so it can adversely affect the results. Secondly, regarding to Table.1, in dimensions less than $1 \mu \mathrm{m}$, Brownian and Phoretic forces are increased severely and can be considered as same level as Buoyancy force. Last but not the least, having furniture in the room, for sure some vortexes have been created which can trap tiny particles. Having little mass, these particles do not have enough momentum to escape from centrifugal force of the vortex so it takes a considerable amount of time for them to get rid of the vortex completely. Moreover, for a better understanding of measured particle concentrations in various diameters, vertical axis of Fig. 1 is plotted logarithmically relative to the horizontal axis.
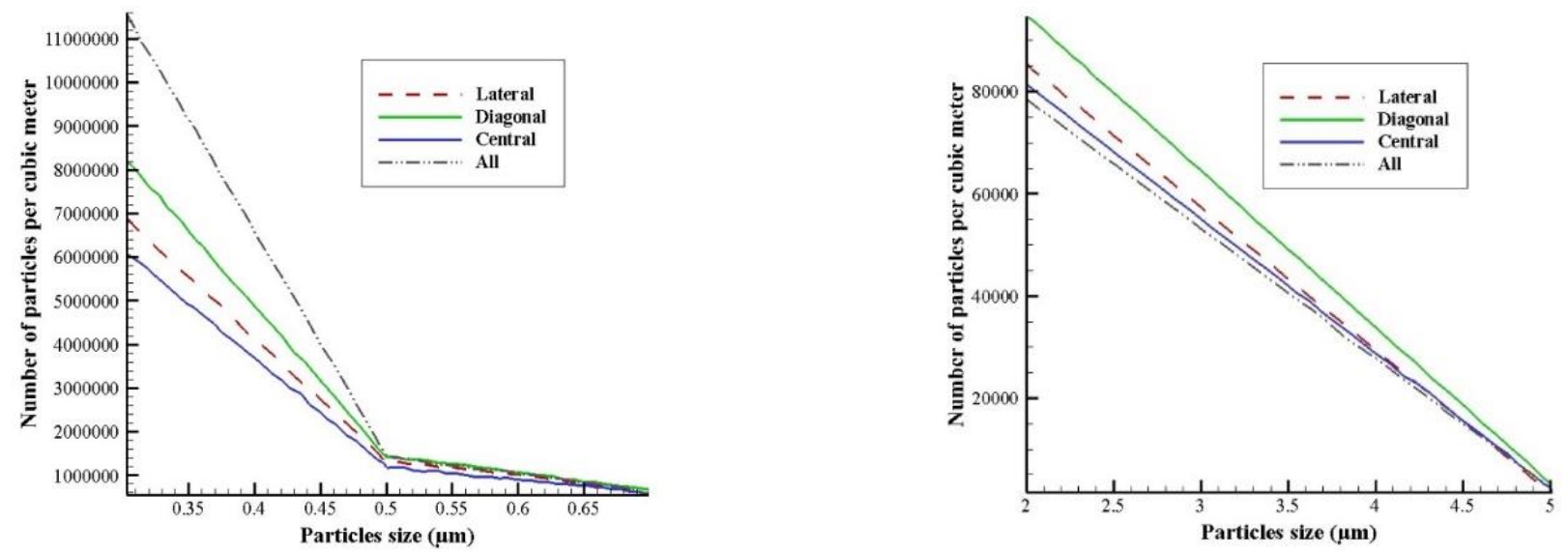

Fig. 1: Particle concentration via Particle size for (a) 0.3 to $0.7 \mu \mathrm{m}$, (b) 2 to $5 \mu \mathrm{m}$ for all arrangements.

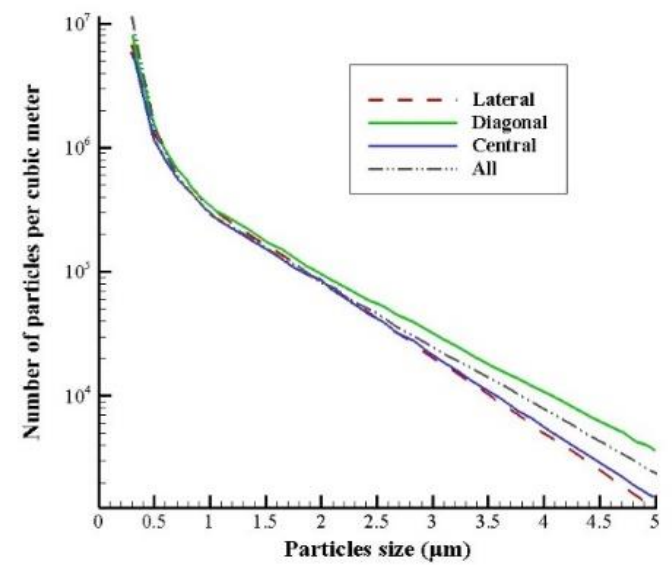

Fig. 2: Logarithmic Particle concentration via Particle size for 0.3 to $5 \mu \mathrm{m}$ for all arrangements.

Generally, numerous factors can lead to the increase of errors and deterioration of the results in experimental studies which can be classified into three different categories. Measuring device error comes first. The flow meter in the study has the accuracy of $0.01 \mathrm{~m} / \mathrm{s}$, however, the inlet velocity is not considered as one of the output parameters of the problem. Also, 
particle counter has the accuracy of $0.3 \mu \mathrm{m}$ which means cannot count particles having dimensions smaller than this value, even though smaller particles are not included in the test. Conspicuously, in smaller dimensions, counting the errors can grow dramatically. Secondly, modeling type error can adversely affect the results. Although the patient, surgeon and some operating tools were used, there are some limitations for exploiting all the equipment and surgical team which are not used completely. Surely, by making the indoor design more complicated, results will become more accurate. Lastly, errors in the measuring method is another trouble. Presence of measuring person in the room, the concentration distribution and stream direction can be adversely affected. Moving velocity, moving direction and position of the measuring person are the main parameters which can directly and effectively influence the indoor stream.

\section{Conclusion}

Noticeably, the full open gates arrangement can remove particles relatively better than the other layouts as it can produce more mass flow rate and more homogeneous pressure gradient in a comparison to the others. However, it may not be practical in reality. In addition, diagonal layout has the worst outlet particle efficiency while the central one has the most. Being mentioned, the expectation was that the lateral arrangement should be the best. Lastly, by decreasing particle dimensions, Buoyancy force will decrease while the Brownian and Phoretic forces will increase to an extent that in dimensions less than $0.5 \mu \mathrm{m}$ results are almost inverted.

\section{References}

[1] D. L. Malone, T. Genuit, J. K. Tracy, C. Gannon, and L. M. Napolitano, "Surgical site infections: reanalysis of risk factors," Journal of Surgical Research, vol. 103, no. 1, pp. 89-95, 2002.

[2] K. B. Kirkland, J. P. Briggs, S. L. Trivette, W. E. Wilkinson, and D. J. Sexton, "The impact of surgical-site infections in the 1990s: attributable mortality, excess length of hospitalization, and extra costs," Infection Control \& Hospital Epidemiology, vol. 20, no. 11, pp. 725-730, 1999.

[3] T. C. Horan, R. P. Gaynes, W. J. Martone, W. R. Jarvis, and T. G. Emori, "CDC definitions of nosocomial surgical site infections, 1992: a modification of CDC definitions of surgical wound infections," Infection Control \& Hospital Epidemiology, vol. 13, no. 10, pp. 606-608, 1992.

[4] S. Y. Tong, J. S. Davis, E. Eichenberger, T. L. Holland, and V. G. Fowler, "Staphylococcus aureus infections: epidemiology, pathophysiology, clinical manifestations, and management," Clinical microbiology reviews, vol. 28, no. 3, pp. 603-661, 2015.

[5] A. Khademi, M. H. Saidi, M. Darbandi, and G. E. Schneider, "Numerical Study of Patient Respiration Effect on Bacterial Dispersion in a Surgery Room," Journal of Fluid Flow, Heat and Mass Transfer (JFFHMT), vol. 6, pp. 4959, 2019.

[6] G. N. Djaiani, M. Ali, L. Heinrich, J. Bruce, J. Carrol, J. Karski, R. J. Cusimano, and D. C. Cheng, "Ultra-fast-track anesthetic technique facilitates operating room extubation in patients undergoing off-pump coronary revascularization surgery," Journal of cardiothoracic and vascular anesthesia, vol. 15, no. 2, pp. 152-157, 2001.

[7] A. Khademi, M. H. Saidi, M. Darbandi, and G. E. Schneider, "Numerical Study of Bacterial Influences Caused by Patient Breathing," in Proceedings of the 6th International Conference of Fluid Flow, Heat and Mass Transfer (FFHMT'19), 2019.

[8] C. A. Balaras, E. Dascalaki, and A. Gaglia, "HVAC and indoor thermal conditions in hospital operating rooms," Energy and Buildings, vol. 39, no. 4, pp. 454-470, 2007.

[9] F. Memarzadeh and A. P. Manning, "Comparison of operating room ventilation systems in the protection of the surgical site/Discussion," ASHRAE transactions, vol. 108, p. 3, 2002.

[10] M. R. Sippola and W. W. Nazaroff, "Particle deposition from turbulent flow: Review of published research and its applicability to ventilation ducts in commercial buildings," Lawrence Berkeley National Lab.(LBNL), Berkeley, CA (United States), 2002. 